\title{
Automatized Estimation of the Effective Thermal Conductivity of Carbon Fiber Reinforced Composite Materials
}

\author{
Zia Javanbakht ${ }^{\mathrm{a}}$, Wayne Hall ${ }^{\mathrm{b}}$, Andreas Öchsner ${ }^{\mathrm{c}}$
}

School of Engineering, Griffith University, Gold Coast Campus, Southport 4222, Australia.

aZia.Javanbakht@gmail.com, bw.hall@griffith.edu.au ,'Andreas.Oechsner@gmail.com

Keywords: Finite element method; Reinforced composites; Thermal conductivity; Carbon nanotubes.

\begin{abstract}
In the current study, the representative volume element (RVE) is used to model randomly generated nanocomposite structures consisting of carbon nanotubes (CNTs) embedded in an epoxy resin matrix. The finite element Method is utilized for numerical simulations and investigation of the influential parameters on the generated RVEs. In order to automatize the whole procedure-from generating the finite element models to conducting the analyses - a subroutine-based programming approach is adopted using the MSC Marc finite element package and Fortran programming language. The simulations can successfully predict the increase in thermal conductivity of CNT-reinforced nanocomposites by increasing the fiber volume fraction.
\end{abstract}

\section{Introduction}

Exceptional physical and mechanical properties of carbon nanotubes have directed a huge amount of research since their first encounter in 1991 [1]. Properties such as high Young's and shear moduli, low density, high thermal conductivity, and ballistic electronic conduction have introduced a wide variety of applications for CNTs in mechanics, electronics, and energy systems among other fields [2]. For instance, some single-walled nanotubes can have elastic modulus and strengths to mass ratios of approximately 19 and 56 times those of typical structural steel, respectively [3]. In terms of their thermal conductivity, values above $2000 \frac{\mathrm{W}}{\mathrm{m} \cdot \mathrm{K}}$ are quite common while extremely high values such as $6600 \frac{\mathrm{W}}{\mathrm{m} \cdot \mathrm{K}}$ have also been reported $[4,5]$.

The limited thermal conductivity of polymers, specially in the amorphous ones, contradicts with their industrial applications, e.g., as heat sinks [5]. This makes polymers preferable mediums for fillers such as CNTs to make up nanocomposites with superior properties. In addition, direct characterization of CNTs is quite limited and thus, to avoid the obstacles of direct measurement of their thermal conductivity, experiments are conducted on the aligned nanotubes which are dispersed in a matrix [6, 7 .

Due to the limitations of the experiments, numerical methods are more and more used for characterising the material properties of nanocomposites. Among these methods, the finite element method is used to simulate various physical and multi-physics phenomena among which are the transfer phenomena. Many recent researches have numerically investigated the effects of fiber aspect ratio [8, 9 , 10], fiber orientation [11, 12], fiber dispersion/agglomeration [13, 14], interface bonding quality [15, 14, 16], waviness/straightness of the fibers [17, 18], fiber volume/weight fraction [19, 20, 21], and property contrast among others. One of the common methods of finite element modelling is using an auxiliary scripting program, such as Python, to generate the mesh (see [22] for instance) whereas in the current study, the effects of random distributions is investigated by an automatised procedure.

\section{Methodology}

The finite element method $[23,24]$ is used in this study to simulate the heat conduction within a composite. The MSC.Marc (version 2014.2) commercial package is selected to carry out the simulations. The package provides a variety of advanced features by means of its Fortran-based subroutines which makes it favorable for customized applications. In addition, the modules provided in [25] are used to facilitated the programming and model generation procedures. 


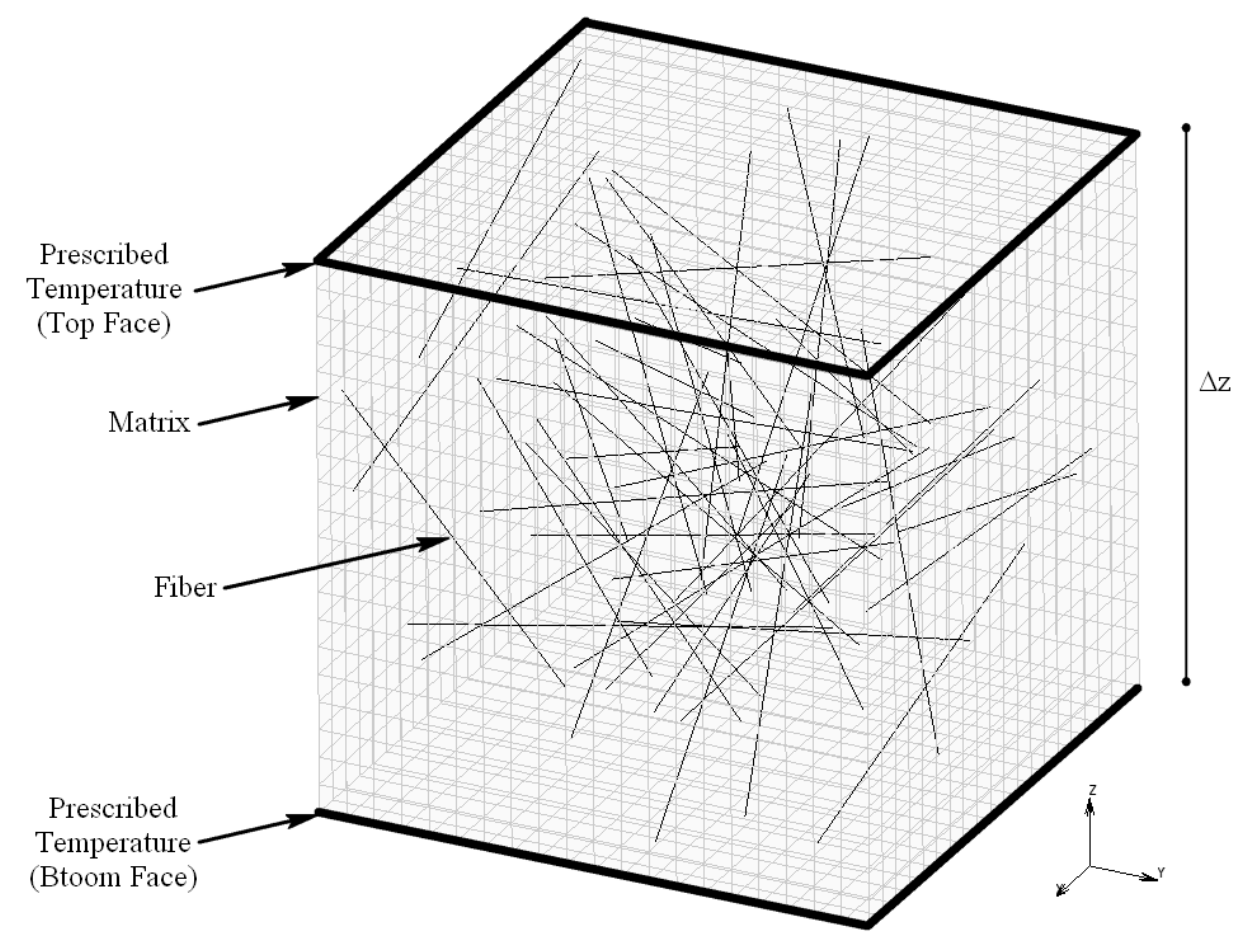

Fig. 1: Meshed RVE with randomly distributed fibers

The other advantage of using Fortran subroutines in the current study is the fact that no other programming languages, e.g. Matlab, is required for generation of the fibers. All the process is carried out using subroutines and thus, at every job submission, a newly generated fiber distribution is incorporated in the mesh. Figure 1 illustrates an instance of dispersed fibers in the representative volume element.

In order to generate the randomly dispersed fibers in the matrix, the MakeFiberNodLst subroutine is used (see Fig. 3). The specified fiber volume fraction and the total number of fibers, i.e., 50 in the current study, are used to calculate the length of each fiber. Then, a loop starts to generate the start and end node coordinates of the fiber. In each cycle, two random coordinates are generated using the GetRandNum() function provided in [25] and the end node coordinates are adjusted to satisfy the length criterion. In addition, it is checked if the the end node is positioned within the RVE or not. If the node is located outside of the RVE, a new set of random coordinates is generated for the end node and the process flows back to adjusting the length of the fiber. Otherwise, the algorithm moves on to the next fiber. The generated fibers are inserter into the matrix and thus, no node matching is required in this procedure (see [26] for more information).

The dimensions of the RVE are $20 \times 20 \times 20 \mathrm{~nm}^{3}$ and the equivalent cross sectional area of the CNTs are $1.13 \mathrm{~nm}^{2}$ which was calculated based on a wall thickness of $0.34 \mathrm{~nm}$, and an outer diameter of $1.4 \mathrm{~nm}$ [27]. The material properties of the matrix and fibers are shown in Table 1.

Two types of heat transfer elements were engaged in the thermal analyses: the 8-node isoparametric

Table 1: Material properties of the composite components

\begin{tabular}{ccc}
\hline Component & Material & $\begin{array}{c}\text { Average conductivity } \\
\left(\frac{\mathrm{W}}{\mathrm{m} \cdot \mathrm{K}}\right)\end{array}$ \\
\hline Matrix & Epoxy resin & $0.214[27]$ \\
Fiber & Carbon nanotube & $2980[28]$ \\
\hline
\end{tabular}

hexahedral elements (type 43) consisted the mesh of the matrix and the straight 2-node link elements (type 36) were used for the fibers. A set of temperature boundary conditions were prescribed for each 


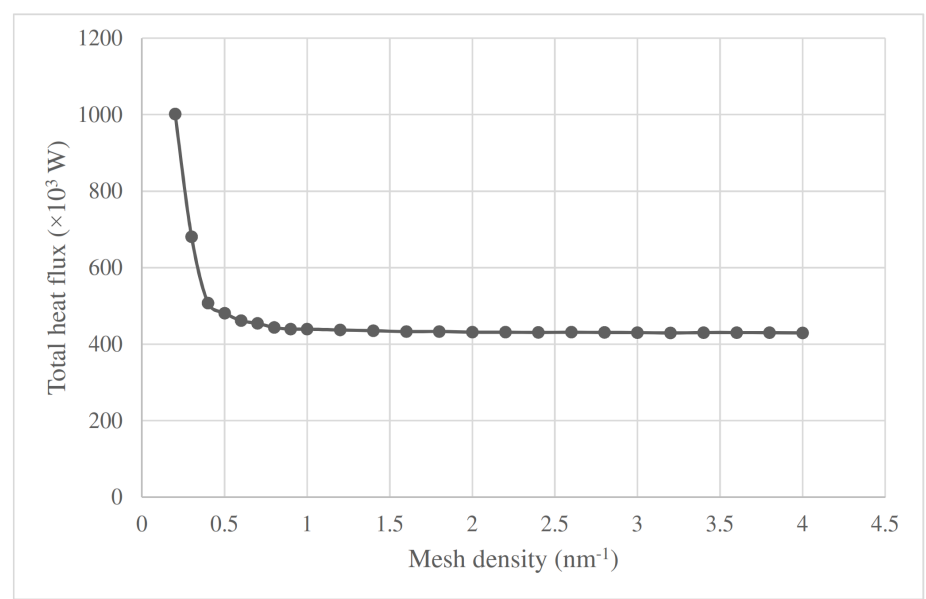

(a) Total heat flux versus mesh density

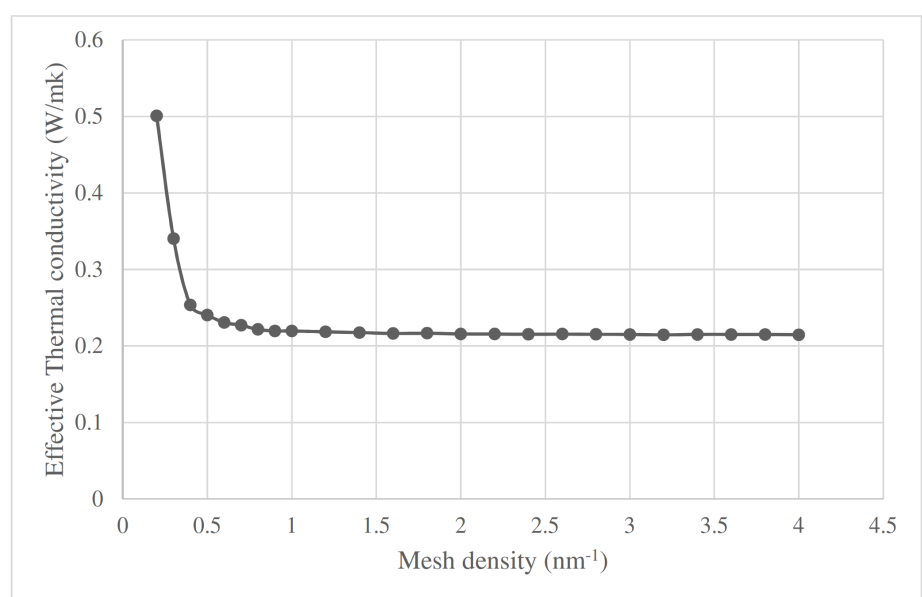

(b) Thermal conductivity versus mesh density

Fig. 2: Results of mesh sensitivity analysis

of the bottom and top face nodes, i.e., constant temperatures zero and 10, respectively. These boundary conditions imposed a temperature gradient in the sample and generated reaction heat fluxes in the aforementioned faces. Finally, the effective thermal conductivity of the composite was calculated using Fourier's law [29]:

$$
\lambda=\frac{\dot{Q}}{A_{0}} \cdot \frac{\Delta z}{\Delta T}
$$

where $\lambda$ is the effective thermal conductivity, $\dot{Q}$ is the total reaction flux, $A_{0}$ is the cross-sectional area along the flux, $\Delta z$ is the distance between the top and bottom faces, and $\Delta T$ is the applied temperature difference.

\section{Results and Discussion}

Since the whole simulation process is automatized, a sensitivity analysis can readily be done. The results for the mesh sensitivity analysis are illustrated in Fig. 2. For a model with only hexahedral cubic elements, the mesh density $\gamma$ can be defined as

$$
\gamma=\frac{1}{a}
$$




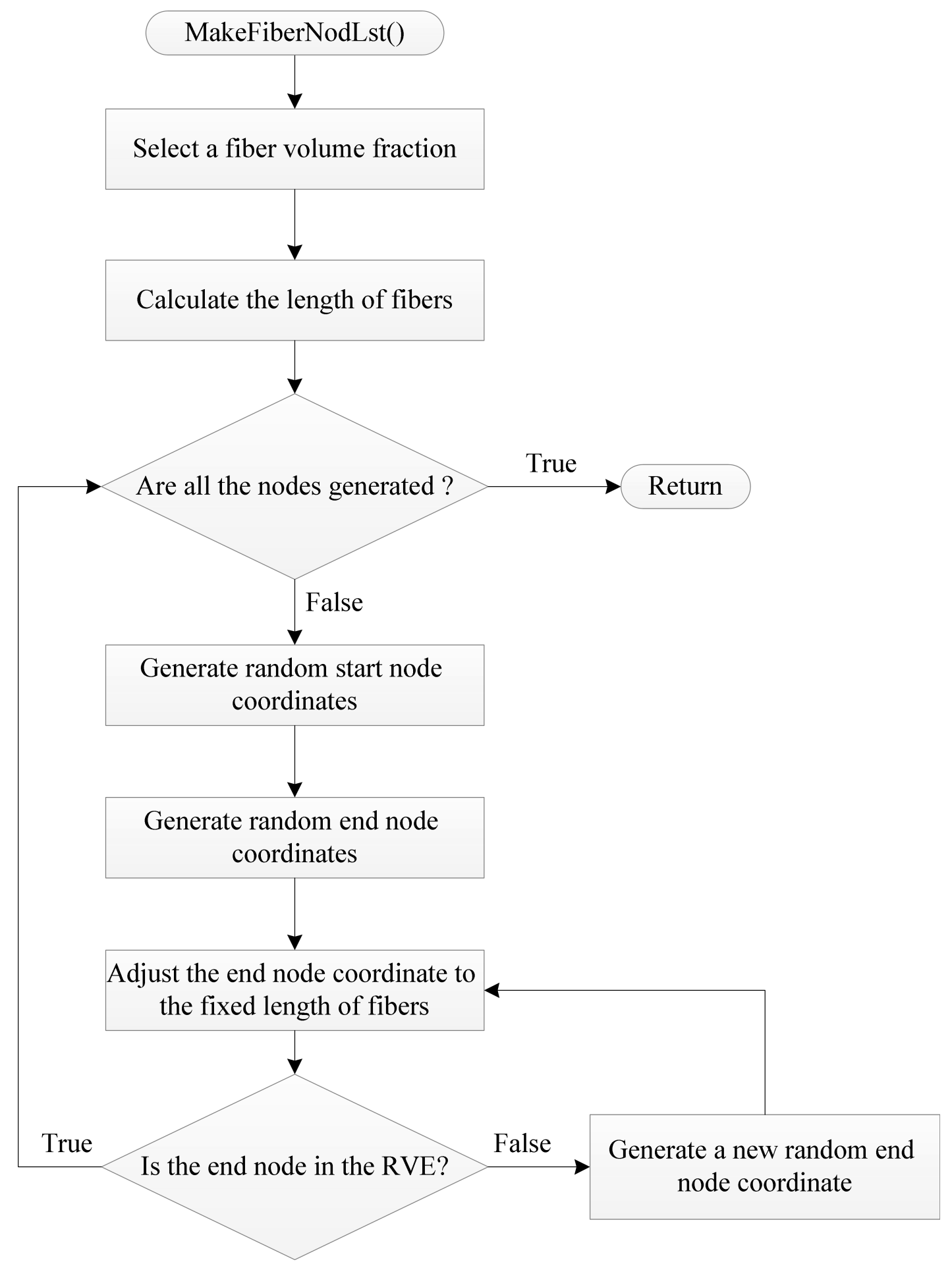

Fig. 3: Flowchart of random coordinate generation for fibers 
where $a$ is a characteristic dimension of the element. In the current study, several mesh densities ranging from 0.2 to 4 have been investigated where a similar rate of convergence was observed for both heat flux and thermal conductivity. Finally, the mesh density of 3 , which corresponds to $0.33 \times$ $0.33 \times 0.33 \mathrm{~nm}$ cubic elements, is selected for the simulations.

The simulations are repeated 10 times for each volume fraction to check the influence of the random fiber generation.

Table 2: Simulation results of thermal conductivity for various fiber volume fractions (10 runs per volume fraction)

\begin{tabular}{ccc}
\hline $\begin{array}{c}\text { Volume fraction } \\
(\%)\end{array}$ & $\begin{array}{c}\text { Average conductivity } \\
\left(\frac{\mathrm{W}}{\mathrm{m} \cdot \mathrm{K}}\right)\end{array}$ & $\begin{array}{c}\text { Standard error } \\
\left(\frac{\mathrm{W}}{\mathrm{m} \cdot \mathrm{K}}\right)\end{array}$ \\
\hline 2.5 & 0.219539 & 0.000119 \\
5.0 & 0.238291 & 0.000907 \\
7.5 & 0.267112 & 0.001446 \\
10 & 0.303779 & 0.003676 \\
\hline
\end{tabular}

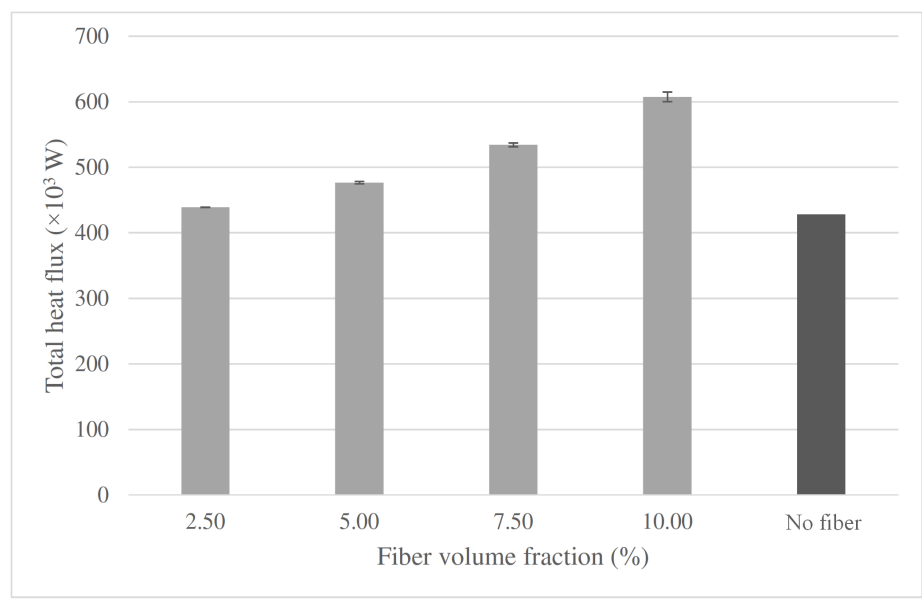

(a) Total heat flux versus various fiber volume fractions

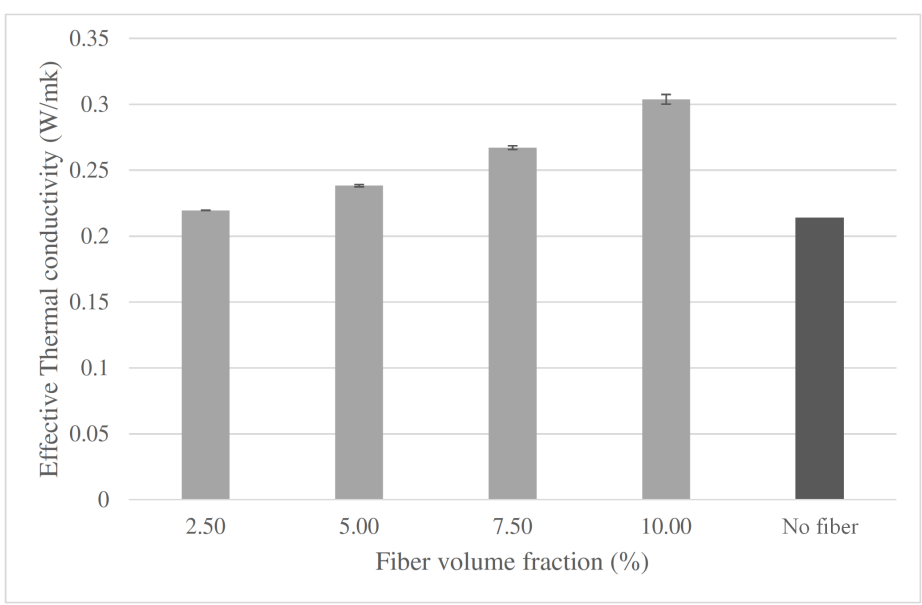

(b) Thermal conductivity versus various fiber volume fractions

Fig. 4: Results of the simulations (average values are based on 10 randomly generated fiber distributions at a constant fiber volume content) 


\section{Conclusions}

In the current study, the effective thermal conductivity of randomly dispersed fibers was investigated. Random fibers were generated in every job submission by means of an automatic procedure which provides more flexibility when several consecutive analyses are required, e.g., a mesh sensitivity analysis. Adding CNTs to the matrix alters the thermal conductivity of the composite compared to the pure matrix material. Namely, by increasing the fiber volume fraction, the effective conductivity of the composite increases. Additionally, the randomness of fiber dispersion in the matrix increases the standard error as the fiber volume fraction increases for a specific number of fibers.

\section{References}

[1] S. Iijima. "Helical microtubules of graphitic carbon". Nature 354.6348 (1991), pp. 56-58.

[2] A. Jorio, G. Dresselhaus, and M. S. Dresselhaus. Carbon nanotubes: Advanced topics in the synthesis, structure, properties and applications. Vol. 111. Topics in applied physics, 03034216. Berlin: Springer-Verlag, 2008.

[3] R. H. Baughman, A. A. Zakhidov, and W. A. de Heer. "Carbon nanotubes-the route toward applications". Science (New York, N.Y.) 297.5582 (2002), pp. 787-792.

[4] S. Berber, Y.-K. Kwon, and D. Tománek. "Unusually High Thermal Conductivity of Carbon Nanotubes". Phys. Rev. Lett. 84.20 (2000), pp. 4613-4616.

[5] Z. Han and A. Fina. "Thermal conductivity of carbon nanotubes and their polymer nanocomposites: A review". Progress in Polymer Science 36.7 (2011), pp. 914-944.

[6] R. S. Ruoff and D. C. Lorents. "Mechanical and thermal properties of carbon nanotubes". Carbon 33.7 (1995), pp. 925-930.

[7] E. T. Thostenson, Z. Ren, and T.-W. Chou. "Advances in the science and technology of carbon nanotubes and their composites: A review”. Composites Science and Technology 61.13 (2001), pp. 1899-1912.

[8] H. R. Lusti and A. A. Gusev. "Finite element predictions for the thermoelastic properties of nanotube reinforced polymers". Modelling and Simulation in Materials Science and Engineering 12.3 (2004), S107-S119.

[9] Z. Shan and A. M. Gokhale. "Representative volume element for non-uniform micro-structure". Computational Materials Science 24.3 (2002), pp. 361-379.

[10] I. E. Afrooz and A. Öchsner. "Effect of the Carbon Nanotube Distribution on the Thermal Conductivity of Composite Materials". Journal of Heat Transfer 137.3 (2015), p. 034501.

[11] N. Khani, M. Yildiz, and B. Koc. "Elastic properties of coiled carbon nanotube reinforced nanocomposite: A finite element study”. Materials \& Design 109 (2016), pp. 123-132.

[12] R. Makvandi and A. Öchsner. "On a numerical strategy to simulate nanotube-reinforced composite materials". Materialwissenschaft und Werkstofftechnik 45.5 (2014), n/a-n/a.

[13] S. R. Bakshi, R. R. Patel, and A. Agarwal. "Thermal conductivity of carbon nanotube reinforced aluminum composites: A multi-scale study using object oriented finite element method". Computational Materials Science 50.2 (2010), pp. 419-428.

[14] H. S. Hedia et al. "Effect of agglomeration and dispersion on the elastic properties of polymer nanocomposites: A Monte Carlo finite element analysis". Materials Testing 58.3 (2016), pp. 269-279.

[15] G. I. Giannopoulos, S. K. Georgantzinos, and N. K. Anifantis. "A semi-continuum finite element approach to evaluate the Young's modulus of single-walled carbon nanotube reinforced composites". Composites Part B: Engineering 41.8 (2010), pp. 594-601. 
[16] S. I. Kundalwal and M. C. Ray. "Effective properties of a novel continuous fuzzy-fiber reinforced composite using the method of cells and the finite element method". European Journal of Mechanics - A/Solids 36 (2012), pp. 191-203.

[17] M. A. Bhuiyan et al. "Understanding the effect of CNT characteristics on the tensile modulus of CNT reinforced polypropylene using finite element analysis". Computational Materials Science 79 (2013), pp. 368-376.

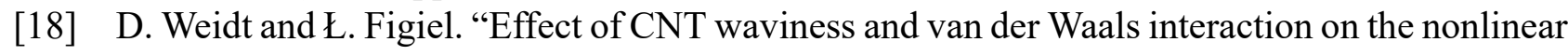
compressive behaviour of epoxy/CNT nanocomposites". Composites Science and Technology 115 (2015), pp. 52-59.

[19] S. I. Kundalwal and M. C. Ray. "Effective properties of a novel composite reinforced with short carbon fibers and radially aligned carbon nanotubes". Mechanics of Materials 53 (2012), pp. 47-60.

[20] K. Alasvand Zarasvand and H. Golestanian. "Determination of nonlinear behavior of multiwalled carbon nanotube reinforced polymer: Experimental, numerical, and micromechanical". Materials \& Design 109 (2016), pp. 314-323.

[21] X. L. Chen and Y. J. Liu. "Square representative volume elements for evaluating the effective material properties of carbon nanotube-based composites". Computational Materials Science 29.1 (2004), pp. 1-11.

[22] A. Jafari, A. A. Khatibi, and M. M. Mashhadi. "Comprehensive investigation on hierarchical multiscale homogenization using Representative Volume Element for piezoelectric nanocomposites". Composites Part B: Engineering 42.3 (2011), pp. 553-561.

[23] A. Öchsner and M. Merkel. One-Dimensional Finite Elements: An Introduction to the FE Method. Berlin and Heidelberg: Springer, 2013.

[24] A. Öchsner. Computational statics and dynamics: An introduction based on the finite element method. Singapore: Springer, 2016.

[25] Z. Javanbakht and A. Öchsner. Advanced Finite Element Simulation with MSC Marc: Application of User Subroutines. 1ST ED. 2017. Springer International, 2017.

[26] MSC Software Corporation. Marc 2014.2: Theory and User Information. Vol. A. Newport Beach, California: MSC Software Corporation, 2014.

[27] R. Makvandi and A. Öchsner. "On a Finite Element Approach to Predict the Thermal Conductivity of Carbon Fiber Reinforced Composite Materials". Defect and Diffusion Forum 354 (2014), pp. 215-225.

[28] T. Fiedler, E. Solórzano, and A. Öchsner. "Numerical and experimental analysis of the thermal conductivity of metallic hollow sphere structures". Materials Letters 62.8-9 (2008), pp. 1204 1207.

[29] T. Fiedler et al. "A refined finite element analysis on the thermal conductivity of perforated hollow sphere structures”. Computational Materials Science 47.2 (2009), pp. 314-319. 